\title{
Two-bridge links with strong triviality
}

\author{
Ichiro TORISU
}

Naruto University of Education

(Communicated by K. Taniyama)

Abstract. In this article, we study strong triviality of two-bridge links. We prove that every (non-trivial) two-bridge link can not be strongly $n$-trivial for $n \geq 1$.

\section{Introduction}

A knot $K$ in $S^{3}$ is called strongly $n$-trivial if there exist $n+1$ crossings contained in a diagram of $K$ such that the result of any $0<m \leq n+1$ crossing changes on these crossings is the trivial knot (Figure 1). Note that by definition, the unknotting number of strongly $n$-trivial knots is one. Recently, Askitas-Kalfagianni and Howards-Luecke have started the study of strongly $n$-trivial knots by using 3-manifold topology. See [1], [3]. In the previous paper [7], the author determined strong triviality of two-bridge knots, that is, only the trivial knot, the trefoil knot and the figure-eight knot have both two-bridge and strongly $n$-trivial diagrams for $n \geq 1$.

In the current paper, we continue to study strong triviality for links. Especially, we prove that every (non-trivial) two-bridge link can not be strongly $n$-trivial for $n \geq 1$.
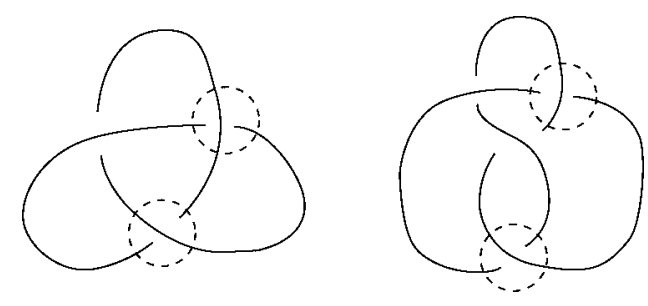

FIGURE 1. Strongly 1-trivial knots.

Received November 6, 2004; revised February 23, 2005

1991 Mathematics Subject Classification. 57M25.

Key words and phrases. strongly $n$-trivial, two-bridge link, $S^{2} \times S^{1}$, Dehn surgery. 

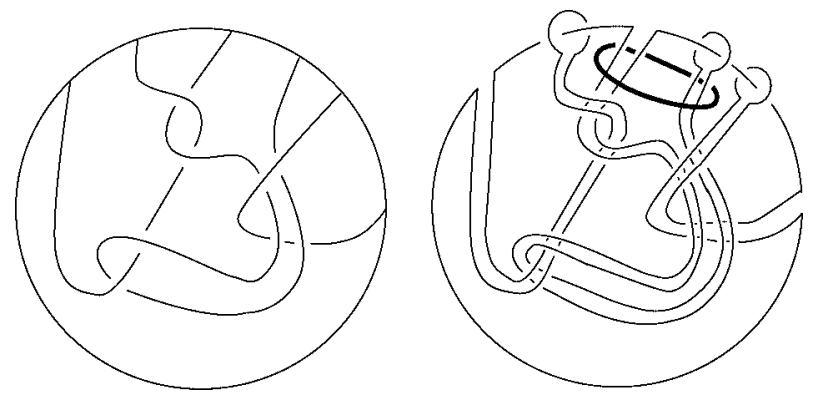

FIGURE 2. A Brunnian Suzuki graph and a strongly 2-trivial link.

\section{Main result}

Definition 2.1. Let $n$ be a non-negative integer. A k-component link $L$ in $S^{3}$ is strongly $n$-trivial if there exist $n+1$ crossings contained in a diagram of $L$ such that the result of any $0<m \leq n+1$ crossing changes on these crossings is the $k$-component trivial link.

We remark that for a strongly $n$-trivial $k$-component link $L$, any crossing change in Definition 2.1 must be done on one component of $L$ and the linking number of any 2-component in $L$ is zero. Moreover Vassiliev invariants for $L$ of orders $\leq n$ coincide with that for the $k$-component trivial link. As in [1], [3], we can construct strongly $n$-trivial knots for any $n$ via "Brunnian Suzuki graphs" as follows. A Brunnian Suzuki graph $G$ consists of one circle $C$ and edges $E$ such that if we remove any non-empty subset of $E$ from $G$, the resulting graph is trivial. By creating hooks along $E$ as indicated in Figure 2, we have a strongly $n$-trivial knot $K$, where $n=\sharp|E|-1(\sharp|\cdot|$ denotes the cardinality). Take a disk $D$ such that (i) $\partial D$ surrounds the ends of one edge in $E$, (ii) $D$ does not intersect other edges in $E$, (iii) $\partial D$ is sufficiently close to $C$, as indicated by a bold circle in Figure 2 . Then we have a strongly $n$-trivial 2-component link $K \cup \partial D$.

Let $S(\alpha, \beta)$ be a two-bridge link whose two-fold branched cover is the lens space $L(\alpha, \beta)$, where $\alpha$ is an even integer and $\beta$ is an integer coprime to $\alpha$. Note that $S(\alpha, \beta)$ is a 2 -component link and $S(0,1)$ is the trivial link.

Our theorem is then the following.

THEOREM 2.2. If a two-bridge link $S(\alpha, \beta)$ is non-trivial, then $S(\alpha, \beta)$ can not be strongly $n$-trivial for every $n \geq 1$.

REMARK 2.3. (i) Two-bridge links with unlinking number one was determined by Kohn in [4]. He proved that a two-bridge link $S(\alpha, \beta)$ has unlinking number one if and only if $\alpha=2 r^{2}$ and $\beta=2 r s \pm 1$ for some coprime integers $r$ and $s$. Actually, the proof of Theorem 2.2 is simiar to that of Theorem 1 in [4].

(ii) After writing this paper, the author was informed that Tsutsumi have proved that strongly $n$-trivial links are boundary links [8]. So Theorem 2.2 is also obtained from his theorem. 

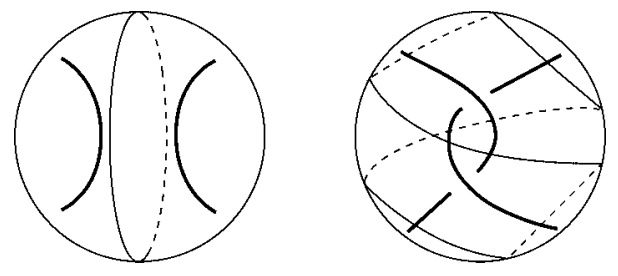

FIGURE 3.

\section{Proof of Theorem 2.2}

Since strongly $n$-trivial link is obviously strongly $m$-trivial for all $m \leq n$, it is sufficient to show that if $S(\alpha, \beta)$ is strongly 1-trivial, then $S(\alpha, \beta)$ is the 2-component trivial link. As in [4], [7], we will prove Theorem 2.2 via Dehn surgery techniques.

Let $N(k)$ be a regular neighbourhood of a knot $k$ in a closed orientable 3-manifold $M$, with $\mu$ a meridian for $N(k)$ and let $E(k)=M-$ int $N(k)$ be the exterior of $k$ in $M$. Now let $k(\gamma)$ denote the manifold obtained by attaching a solid torus $V$ to $E(k)$ so that a curve of slope $\gamma$ on $\partial E(k)$ bounds a disk in $V$, where $\gamma$ indicates the isotopy class of an essential simple closed curve on $\partial E(k)$. For a knot $k$ in $S^{3}$, a slope $\gamma$ is represented by an element $\frac{m}{n} \in \mathbf{Q} \cup\left\{\frac{1}{0}\right\}$, where $\gamma=m \mu_{k}+n \lambda_{k}$ in $H_{1}(\partial E(k), \mathbf{Z}),\left(\mu_{k}, \lambda_{k}\right)$ is a preferred meridian-longitude pair of $k$ and $m, n$ are coprime integers. We shall say that $k(\gamma)$ is the result of $\gamma$-Dehn surgery on $k$. Dehn surgery on a 2-component link $k \cup k^{\prime}$ is also defined in the same way and denoted by $k \cup k^{\prime}\left(\gamma, \gamma^{\prime}\right)$ for slopes $\gamma$ and $\gamma^{\prime}$ of $k$ and $k^{\prime}$, respectively. For two slopes $\gamma$ and $\delta$ in $\partial E(k)$, let $\Delta(\gamma, \delta)$ be their minimal geometric intersection number.

The next well-known lemma was first observed by Montesinos in [5].

LEMMA 3.1. Let $K$ and $K^{\prime}$ be knots in $S^{3}$ and let $M_{K}$ and $M_{K^{\prime}}$ be the two-fold branched covering spaces of $S^{3}$ along $K$ and $K^{\prime}$, respectively. Suppose the result of a crossing change on $K$ is $K^{\prime}$. Then $M_{K^{\prime}}$ is obtained by $\gamma$-Dehn surgery on some knot $k$ in $M_{K}$, with $\Delta(\gamma, \mu)=2$.

PROOF. We regard the crossing change as a replacement of sufficiently small 2-string trivial tangles $(B, t) \hookrightarrow\left(S^{3}, K\right)$ and $\left(B, t^{\prime}\right) \hookrightarrow\left(S^{3}, K^{\prime}\right)$. Then the two-fold branched covering spaces of the tangles along the 2-strings are solid tori $V$ and $V^{\prime}$, respectively. Therefore $M_{K^{\prime}}$ equals the result of some $\gamma$-Dehn surgery on a knot in $M_{K}$. Let $D$ and $D^{\prime}$ be essential meridian disks for $(B, t)$ and $\left(B, t^{\prime}\right)$, respectively (see Figure 3 ). Then in $M_{K}$, the preimages of each component of $\partial D$ and $\partial D^{\prime}$ essentially intersects each other at 2 points on $\partial V=\partial V^{\prime}$. Hence we have $\Delta(\gamma, \mu)=2$.

A torus knot in a lens space is a knot isotopic onto a Heegaard torus of the lens space. For the proof of Theorem 2.2, we need the following theorem essentially obtained in [6]. 
THEOREM 3.2. Let $k_{T}$ be a torus knot in $S^{2} \times S^{1}$. Suppose that $k_{T}(\gamma)$ is homeomorphic to $S^{2} \times S^{1}$ for some slope $\gamma$ with $\Delta(\gamma, \mu)=2$. Then $k_{T}$ is the trivial knot, that is, $k_{T}$ bounds a disk in $S^{2} \times S^{1}$.

Proof. We use the notation in [6, p. 1568] and suppose that $k_{T}$ is a " $(m, n)$-torus knot" $C_{m, n}$ in $L(0,1)=S^{2} \times S^{1}$, where $m$ and $n$ are coprime integers. In this case, remark that $C_{m, n}$ is trivial if and only if $n=0$. Then by [6, Lemma 7], if $k_{T}(\gamma)$ is a lens space, then $k_{T}(\gamma)$ is orientation preserving homeomorphic to $L\left(-2 n^{2}, 2 b n^{2}+2 m n \pm 1\right.$ ) for some $b$. (In [6, p. 1568], $r=0, s=1, a=-1, d=2$ ). Therefore by our assumption, $n$ must be zero. This completes the proof.

The next easy lemma depends on a specific property of $S^{2} \times S^{1}$.

LEMMA 3.3. A torus knot in $S^{2} \times S^{1}$ represents the trivial element in the fundamental group of $S^{2} \times S^{1}$, which is isomorphic to $\mathbf{Z}$, if and only if it is the trivial knot.

PROPOSITION 3.4. If a two-bridge link $S(\alpha, \beta)$ is strongly 1-trivial, then there is a 2component link $k_{1} \cup k_{2}$ in $S^{2} \times S^{1}$ such that $k_{1}\left(\gamma_{1}\right)$ and $k_{2}\left(\gamma_{2}\right)$ are homeomorphic to $S^{2} \times S^{1}$ and $k_{1} \cup k_{2}\left(\gamma_{1}, \gamma_{2}\right)$ is homeomorphic to $L(\alpha, \beta)$, where each slope $\gamma_{i}$ satisfies $\Delta\left(\gamma_{i}, \mu\right)=2$. Moreover each $k_{i}$ is the trivial knot in the original $S^{2} \times S^{1}$ and a torus knot in $k_{j}\left(\gamma_{j}\right)=S^{2} \times S^{1}$ $(i \neq j)$.

PROOF. By definition, there are two crossings in a diagram of $S(\alpha, \beta)$ such that both each crossing change and simultaneous crossing changes yield the 2-component trivial link. The two-fold cover of the 2-component trivial link in $S^{3}$ is $S^{2} \times S^{1}$. Therefore by Lemma 3.1 and its proof, there exists a 2-component link $k_{1} \cup k_{2}$ in $S^{2} \times S^{1}$ such that $k_{1}\left(\gamma_{1}\right)=k_{2}\left(\gamma_{2}\right)=$ $S^{2} \times S^{1}$ and $k_{1} \cup k_{2}\left(\gamma_{1}, \gamma_{2}\right)=L(\alpha, \beta)$, where $\Delta\left(\gamma_{i}, \mu\right)=2(i=1,2)$. Moreover, since $\Delta\left(\gamma_{i}, \mu\right)=2$, by Culler-Gordon-Luecke-Shalen's cyclic surgery theorem in [2], the exterior spaces $E\left(k_{1}\right)$ and $E\left(k_{2}\right)$ are Seifert fibred manifolds, respectively. But as in [6, Lemma 4] this implies $k_{1}$ and $k_{2}$ are fibers for some Seifert fibrations of both $S^{2} \times S^{1}$ and $L(\alpha, \beta)$. Moreover, it is well-known that any fiber for a Seifert fibration of a lens space is isotopic to some torus knot (for example, see [6]). By Theorem 3.2, it follows that $k_{1}$ and $k_{2}$ are trivial in the original $S^{2} \times S^{1}$, respectively. This completes the proof of Proposition 3.4.

We are ready to prove Theorem 2.2.

Proof of TheOREM 2.2. Suppose a two-bridge link $S(\alpha, \beta)$ is strongly 1-trivial. Then there is a 2-component link $k_{1} \cup k_{2}$ in $S^{2} \times S^{1}$ which satisfies the properties in the statement of Proposition 3.4. Since $k_{2}$ is trivial, $k_{1}$ in $k_{2}\left(\gamma_{2}\right)=S^{2} \times S^{1}$ may be considered as a full-twisted $k_{1}$ along some disk spanned by $k_{2}$ in the original $S^{2} \times S^{1}$. Therefore notice that the full-twisted $k_{1}$ is also homotopic to zero. By our assumption and Lemma 3.3, we conclude that the full-twisted $k_{1}$ is the trivial knot in $S^{2} \times S^{1}$. It is well-known that Dehn surgery on the trivial knot in $S^{2} \times S^{1}$ is either a non-trivial connected sums of two lens spaces or $S^{2} \times S^{1}$ 
itself. Hence it follows that $k_{1} \cup k_{2}\left(\gamma_{1}, \gamma_{2}\right)=L(\alpha, \beta)$ is equal to $S^{2} \times S^{1}$. So we have $\alpha=0$ and $\beta=1$ and $S(\alpha, \beta)=S(0,1)$ is the 2-component trivial link in $S^{3}$.

This completes the proof of Theorem 2.2.

\section{References}

[1] N. Askitas-E. Kalfagianni, On knot adjacency, Topology and its Appl. 126 (2002), 63-81.

[2] M. Culler-C. Gordon-J. Luecke-P. Shalen, Dehn surgery on knots, Ann. of Math. (2) 125 (1987), 237-300.

[3] H. HowARDS-J. LuECKE, Strongly $n$-trivial knots, Bull. London Math. Soc. 34 (2002), 431-437.

[4] P. Kohn, Two-bridge links with unlinkng number one, Proc. Amer. Math. Soc. 113 (1991), 1135-1147.

[5] J. M. Montesinos, Surgery on links and double branched coverings of $S^{3}$, Ann. of Math. Studies 84 (1975), $227-259$.

[6] I. TORISU, The determination of the pairs of two-bridge knots or links with Gordian distance one, Proc. Amer. Math. Soc. 126 (1998), 1565-1571.

[7] I. TORISU, On strongly $n$-trivial 2-bridge knots, Math. Proc. Cambridge Philos. Soc. 137 (2004), 613-616

[8] Y. Tsutsumi, Strongly $n$-trivial links are boundary links, preprint.

Present Address:

NARUTO UNIVERSITY OF EDUCATION,

NARUTO-CHO, NARUTO-SHI, 772-8502 JAPAN.

e-mail: torisu@naruto-u.ac.jp 